\title{
Isolation and Culture of Single Myofiber and Immunostaining of Satellite Cells from Adult C57BL/6J Mice \\ Shujie Chen ${ }^{1, \#}$, Hongrong Ding ${ }^{1, \#}$, Xiangping Yao ${ }^{1,2, \#}$ and Liwei Xie ${ }^{1,2, *}$
}

\author{
${ }^{1}$ State Key Laboratory of Applied Microbiology Southern China, Guangdong Provincial Key Laboratory \\ of Microbial Culture Collection and Application, Guangdong Open Laboratory of Applied Microbiology, \\ Guangdong Institute of Microbiology, Guangdong Academy of Sciences, Guangdong 510070, China; \\ ${ }^{2}$ Zhujiang Hospital, Southern Medical University, Guangdong 510282, China \\ *For correspondence: xielw@gdim.cn \\ ${ }^{\#}$ Contributed equally to this work
}

[Abstract] Myofiber isolation followed with ex vivo culture could recapitulate and visualize satellite cells (SCs) activation, proliferation, and differentiation. This approach could be taken to understand the physiology of satellite cells and the molecular mechanism of regulatory factors, in terms of the involvement of intrinsic factors over SCs quiescence, activation, proliferation and differentiation. Single myofiber culture has several advantages that the traditional approach such as FASC and cryosection could not compete with. For example, myofiber isolation and culture could be used to observe SCs activation, proliferation and differentiation at a continuous manner within their physiological "niche" environment while FACS or cryosection could only capture single time-point upon external stimulation to activate satellite cells by $\mathrm{BaCl}_{2}$, Cardiotoxin or ischemia. Furthermore, in vitro transfection with siRNA or overexpression vector could be performed under ex vivo culture to understand the detailed molecular function of a specific gene on SCs physiology. With these advantages, the physiological state of SCs could be analyzed at multiple designated time-points by immunofluorescence staining. In this protocol, we provide an efficient and practical protocol to isolate single myofiber from EDL muscle, followed with ex vivo culture and immunostaining.

Keywords: Myofiber isolation and culture, Muscle satellite cells, Immunofluorescence staining

[Background] Satellite cells are considered as an adult stem cell because they maintain self-renew and remarkable postnatal regenerative potential of skeletal muscle (Collins et al., 2005). SCs are located between the basal lamina and the plasma lemma of myofibers (MAURO, 1961). Here, to isolate the single myofiber, EDL muscle is digested with the collagenase to release the connective tissue and completely dissociate the connective tissue between fibers. In 1986, it was reported that the proliferation of satellite cells on single muscle fibers were isolated from adult rats and were cultured in cell culture plate (Bischoff, 1986). It was further developed into a tissue culture system that reliably permits isolation of intact, living, single muscle fibers with associated satellite cells from predominantly fast- and slowtwitch muscles of rat or mouse (Bekoff and Betz, 1977a and 1977b; Rosenblatt et al., 1995). There are two main approaches to study SCs: single myofiber culture and primary myoblast culture prepared from mononucleated cells dissociated from whole muscle (Danoviz and Yablonka-Reuveni, 2012). Even 
though, primary myoblast could be split and passaged multiple times, these primary myoblasts retain in proliferation or differentiation states. Freshly isolated single myofiber allows satellite cells to stay beneath the basal lamina at quiescent state, followed with activation by either internal environment or external factors. There are several improvements for myofiber isolation and culture in recent years (Danoviz and Yablonka-Reuveni, 2012; Pasut et al., 2013; Gallot et al., 2016; Lim et al., 2018). However, the difficulty of myofiber isolation and ex vivo culture prevent further application of this reliable and practical method. In the current protocol, we optimized reagents used for single myofiber isolation and improved procedures to make it even more simple and easy to repeat from hand to hand. Since satellite cells are sensitive to various factors, we describe a relatively simple, detailed and efficient approach to isolate and culture single myofiber. Based on our protocol, state and function of satellite cells could be analyzed from mice with different genotypes. Different manipulation such as transfection, and drug treatment on myofibers, followed with the downstream procedures including but not limited to myofiber transplantation and immunostaining could be performed. These additional manipulations could not be performed in these approaches such as FASC or cryosection.

\section{Materials and Reagents}

1. Pipette tips (Jet Biofil, catalog number: PMT950200)

2. $15 \mathrm{ml}$ tube (TrueLine, catalog number: TR2011)

3. $60 \mathrm{~mm}$ Petri Dish, Sterile (Jet Biofil, catalog number: 62060-60)

4. 24-well Plates, Sterile (Corning, Costar, catalog number: CLS3524-100EA)

5. Glass Pasteur Pipettes (Thermo Fisher, catalog number: 1367820A) (Figure 1e-g)

6. Rubber bulbs (Thermo Fisher, catalog number: 1951F15) (Figure 1h)

7. Diamond Pen (XGE, used to cut glass Pasteur pipettes) (Figure 1d)

8. $1 \mathrm{ml}$ Transfer Pipette, sterile (Jet Biofil, catalog number: 25001)

9. Syringe Filters (PTFE, $0.22 \mu \mathrm{m}, 30 \mathrm{~mm}$, Sterile) (Jet Biofil, catalog number: 29525)

10. Microscope glass slide (CITOTEST, catalog number: 70179000)

11. Microscope cover slide $(24 \times 50 \mathrm{~mm})$ (CITOTEST, category number: $10212450 \mathrm{C}$ )

12. Adult C57BL/6J mice at 8-10-week old

13. Glycine (Sangon Biotech, catalog number: A610235, storage temperature: room temperature)

14. Phosphate buffered saline (PBS, Sigma-Aldrich, catalog number: P5368-10PAK, storage temperature: room temperature)

15. Triton X-100 (Sangon Biotech, catalog number: A600198, storage temperature: room temperature)

16. Tween-20 (Sangon Biotech, catalog number: A100777, storage temperature: room temperature)

17. Horse serum (short term storage: $4{ }^{\circ} \mathrm{C}$; long term storage: $-20{ }^{\circ} \mathrm{C}$ ) (Thermo Fisher, catalog number: 16050122)

18. Fetal bovine serum (short term storage: $4^{\circ} \mathrm{C}$; long term storage: $-20^{\circ} \mathrm{C}$ ) (Trinity, catalog number: 010101) 
19. Gibco ${ }^{\mathrm{TM}}$ Sodium Pyruvate $100 \mathrm{mM}$ Solution (Life Technologies, catalog number: 11360070 , storage temperature: $4-8^{\circ} \mathrm{C}$ )

20. Gibco ${ }^{\mathrm{TM}}$ Penicillin-Streptomycin, liquid (Life Technologies, catalog number: $15140-122$, storage temperature: $4-8^{\circ} \mathrm{C}$ )

21. Ethylene Diamine Tetraacetic Acid (EDTA, Solarbio, catalog number: E8040, storage temperature: $4-8{ }^{\circ} \mathrm{C}$ )

22. Gibco ${ }^{\mathrm{TM}}$ Dulbecco's modified Eagle medium (Life Technologies, catalog number: C11995500BT, storage temperature: $4-8{ }^{\circ} \mathrm{C}$ )

23. Bovine Serum Albumin (IgG-Free, Protease-Free, Jackson ImmunoResearch, catalog number: 000-001-162, storage temperature: $4-8^{\circ} \mathrm{C}$ )

24. Fluoroshield ${ }^{\mathrm{TM}}$ with DAPI (Sigma-Aldrich, catalog number: F6057, storage temperature: $4-8{ }^{\circ} \mathrm{C}$ )

25. Primary antibody anti-MyoD (Sigma-Aldrich, catalog number: M6190, storage temperature: 4$\left.8^{\circ} \mathrm{C}\right)$

26. Primary antibody anti-Pax7 (mouse) (Developmental Studies Hybridoma Bank, catalog number: pax 7 , storage temperature: $\left.4-8{ }^{\circ} \mathrm{C}\right)$

27. Secondary antibodies:

a. Goat anti-Rabbit IgG2a Alexa Fluor ${ }^{\circledR} 488$ (Thermo Fisher Scientific, catalog number: A21131, $\left.4-8^{\circ} \mathrm{C}\right)$

b. Goat anti-Mouse IgG Alexa Fluor ${ }^{\circledR} 546$ (Thermo Fisher Scientific, catalog number: A-21123, $\left.4-8^{\circ} \mathrm{C}\right)$

28. Collagenase Type 1 (Worthington Biochemical, catalog number: LS004194, storage temperature: -20 to $-30^{\circ} \mathrm{C}$ )

29. Paraformaldehyde (Sigma-Aldrich, catalog number: P6148, storage temperature: $4-8^{\circ} \mathrm{C}$ )

30. Goat serum (Thermo Fisher, catalog number: 31872 , storage temperature: -20 to $-30{ }^{\circ} \mathrm{C}$ )

31. EDTA (Solarbio, catalog number: E8040)

32. Chicken embryo extract (C3999, Biomol)

33. Washing media (see Recipe 1)

34. Collagenase solution (see Recipe 2)

35. 4\% Paraformaldehyde (see Recipe 3)

36. Culture media (see Recipe 4)

37. Blocking buffer (see Recipe 5)

\section{Equipment}

1. Pipettes (Thermo scientific, Finnpipette F3)

2. Surgical instruments (including a pair of scissors, a pair of fine scissors and a tweezer) (Figure $1 \mathrm{a}, 1 \mathrm{~b}$ and $1 \mathrm{c})$

3. Cell culture hood (AIRTECH, model: BSC-1004IIA2)

4. Mice dissecting table 
5. $4{ }^{\circ} \mathrm{C}$ fridge

6. Centrifuge (Thermo Fisher, catalog number: 75002420)

7. Water bath (DK-8AX)

8. Stereoscopic microscope (Mshot, OLYMPUS SZ61)

9. Heating pad (DeiuxeHeart Mat, model: MDH10)

10. $\mathrm{CO}_{2}$ incubator (Thermo Fisher Scientific, model: 371)

11. Laser scanning confocal microscope (Carl Zeiss, model: LSM 700)

\section{Procedure}

A. Preparation

1. Cut the Glass Pasteur Pipettes (Figure 1e) to a small curved (Figure 1f) and a large (Figure 1g) pipette with a diamond pen (Figure 1d). Polish the terminal with an ethanol flame to smooth the edge. Coat pipettes with undiluted horse serum.

2. Coat two $6-\mathrm{cm}$ cell culture dishes ( $2 \mathrm{ml}$ for each dish) and a 24 -well plate $(400 \mu \mathrm{l}$ for each well) with undiluted horse serum until the entire bottom of plates was covered, followed with incubation for 1 min under a cell culture hood, and then transfer the horse serum back to the original tube and store in a $4{ }^{\circ} \mathrm{C}$ fridge.

3. Uncover the plates and air-dry under a cell culture hood for $1 \mathrm{~h}$.

4. Cover the plates and keep them at room temperature. It is better to coat the plate on the same day of the experiment.

5. Set the temperature of a water bath to $37^{\circ} \mathrm{C}$.

6. Prepare washing media (following Recipe 1 and then keep it in a $37^{\circ} \mathrm{C}$ water bath). In general, $50 \mathrm{ml}$ washing media is enough for up to 200 myofiber isolation from one EDL muscle.

7. Prepare collagenase solution (following Recipe 2 and then keep it in a $37^{\circ} \mathrm{C}$ water bath). Each EDL requires $\sim 1 \mathrm{ml}$ dilated collagenase solution (final concentration: $0.2 \% \mathrm{w} / \mathrm{v}$ ) in a $15 \mathrm{ml}$ tube.

8. Thaw the $4 \%$ paraformaldehyde (following Recipe 3 ).

9. Plug in the heating pad. 


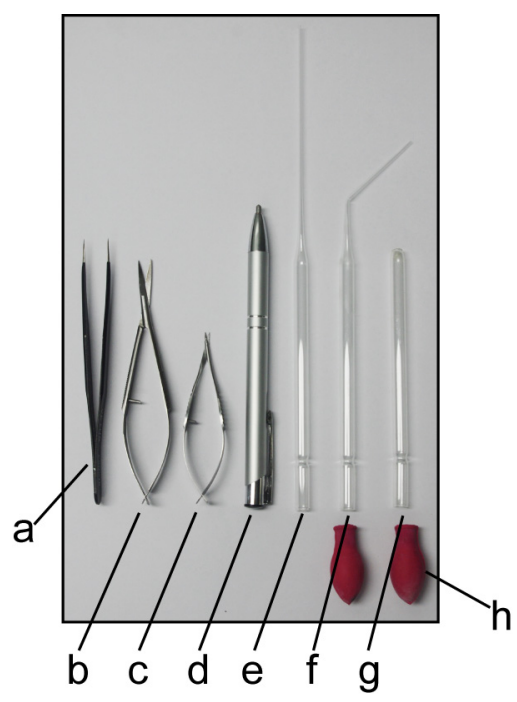

Figure 1. Instruments used for EDL muscle dissection and myofiber isolation. a. Tweezer; b. Scissor; c. Fine scissor; d. Diamond pen; e. Glass Pasteur Pipette; f. Small curved pipette; g. Large pipette; h. Rubber bulbs.

B. EDL Muscle Dissection (Video 1)

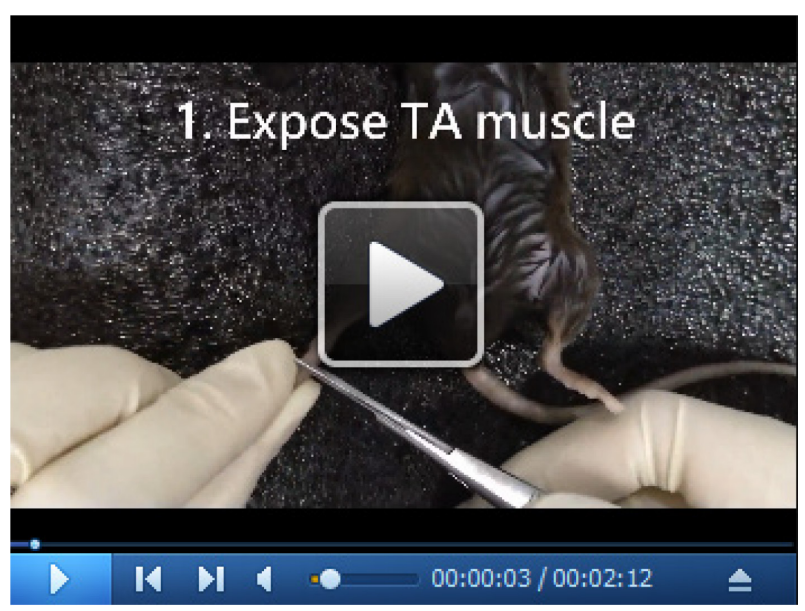

Video 1. Procedures to isolate EDL muscle. This video was made at Guangdong Institute of Microbiology according to guidelines from the Guangdong Institute of Microbiology on Animal Care and approved by the Animal Research Ethics Board of Guangdong Institute of Microbiology under protocol [GT-IACUC201704071].

1. Anesthetize the experimental mice with $\mathrm{CO}_{2}$.

2. Cut the skin below the ankle.

3. Cut through the skin along the inner leg.

4. Expose the muscle fascia and patella by pulling the skin up (Figure 2A).

5. Tore the tibialis anterior (TA) muscle fascia with a tweezer.

6. Tear off vastus lateralis over the knee, expose and cut off the origin of TA and extensor digitorum 
longus (EDL) muscle (Figure 2B).

7. Separate the tendon of TA muscle using a tweezer along the inner margin of the shank. Snip the tendons with the fine scissor (Figure 2C). Gently pull it up to separate the TA muscle from the bone and expose the entire EDL muscle (Figure 2D).

8. Cut the tendon of EDL muscle by snipping two-thirds of the lower ankle, Use one tip of the forceps to separate EDL muscle gently (Figure $2 \mathrm{E}$ ).

9. Place the EDL muscle in a $15 \mathrm{ml}$ tube with the prewarmed collagenase solution and place it in a $37^{\circ} \mathrm{C}$ water bath to incubate for 60 to $80 \mathrm{~min}$ until myofibers are loose but not in crimped (it depends on the efficacy of collagenase and conditions of mice).

10. Dissect the other EDL muscle following Steps B1-B9.
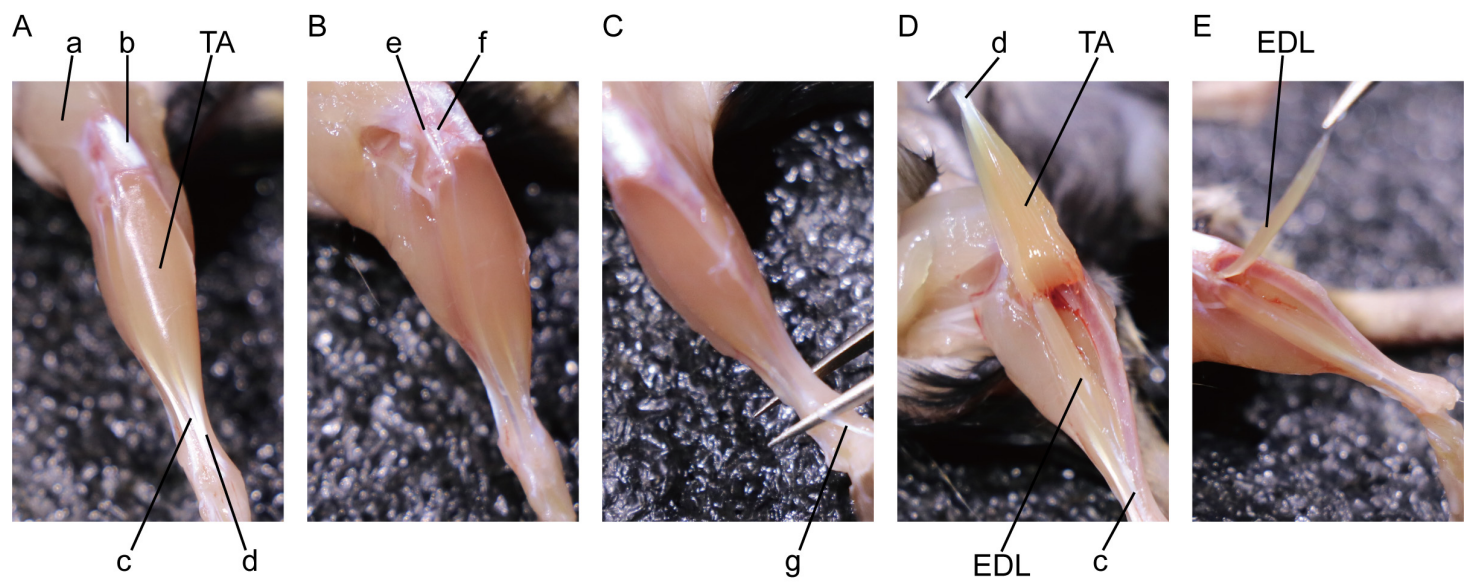

Figure 2. Procedures to isolate the EDL muscle. Pictures presented here depict (A) TA muscle after the skin was removed, (B) Origin of TA and EDL muscle, (C) Distal tendon of TA muscle, (D) EDL muscle after TA muscle was removed, (E) EDL muscle. a. vastus lateralis; b. patella; c. tendon of EDL muscle; d. tendon of TA muscle; e. origin of TA muscle; f. origin of EDL muscle; $g$. tendon of TA muscle.

C. Single Myofiber Isolation (Video 2) 


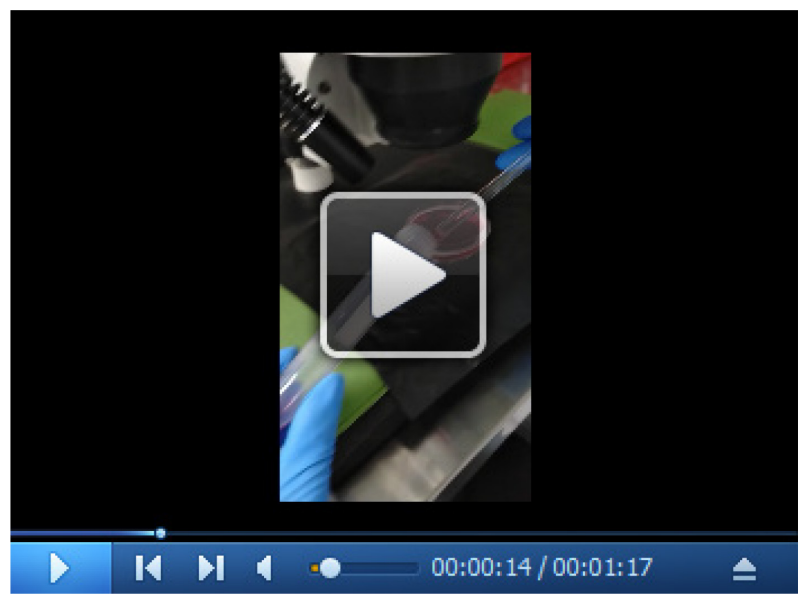

Video 2. Procedures to isolate myofiber from EDL muscle. This video was made at Guangdong Institute of Microbiology according to guidelines from the Guangdong Institute of Microbiology on Animal Care and approved by the Animal Research Ethics Board of Guangdong Institute of Microbiology under protocol GT-IACUC201704071.

1. Transfer the digested EDL muscle to a horse serum coated 6-cm Petri dish with $5 \mathrm{ml}$ prewarmed washing medium in it using the prepared big Glass Pasteur Pipette.

2. Place the 6-cm Petri dish on the heating pad. Inhale an appropriate amount of solution from the dish. Hold the Petri dish with the right hand to adjust the position of dish. Flush the EDL muscle continuously to rotate it along its long pivots until myofibers are naturally released from EDL muscle.

3. When a single muscle fiber is released, use a small bent glass Pasteur pipette to transfer it into a well of 24-well plate covered with $200 \mu$ l washing media. Repeat Steps C1 and C2 until the number of myofibers meets your requirement. It is recommended that the myofibers be collected within 1-h.

4. Wash each well with $500 \mu \mathrm{l}$ washing media three times before adding $500 \mu \mathrm{l}$ culture media (following Recipe 4).

5. Place the 24-well plate into a $\mathrm{CO}_{2}$ incubator at $37^{\circ} \mathrm{C}$.

D. Fixation

1. When myofibers are cultured for 0/24/48/72-h or other different designated length of time, remove as much culture media as possible (leave the minimum amount of culture media to avoid myofibers' exposure to the air).

2. Fix myofibers with $500 \mu \mathrm{l} \mathrm{4 \%} \mathrm{PFA} \mathrm{for} 10 \mathrm{~min}$ at room temperature.

3. Aspirate the fixation buffer, and add $500 \mu \mathrm{l} 50 \mathrm{mM}$ glycine to incubate for $10 \mathrm{~min}$ to quench the fixation at room temperature.

4. Wash myofibers with PBS for 3 times at room temperature. The fixed myofibers can be stored in a $4^{\circ} \mathrm{C}$ fridge for up to a week before immunostaining (Figure 3 ). 


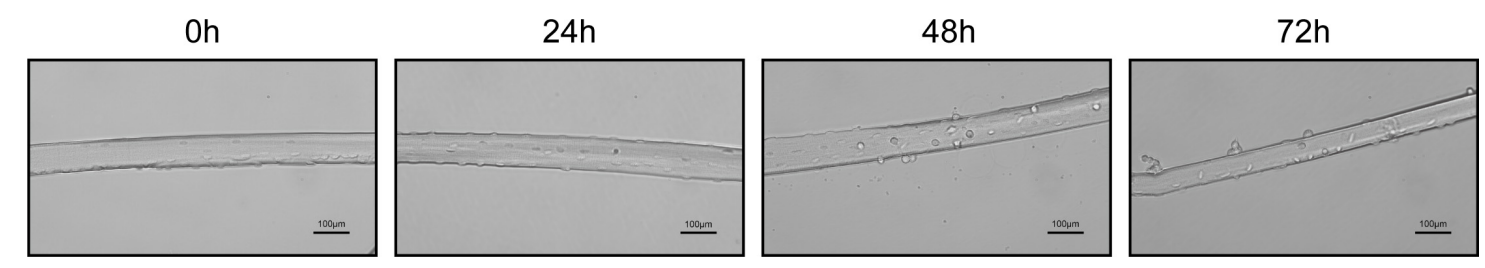

Figure 3. Images of isolated single myofiber after cultured for 0/24/48/72 $\mathrm{h}$. Scale bars = $100 \mu \mathrm{m}$.

E. Immunostaining

1. Permeabilize myofibers with Triton $\mathrm{X}-100(0.5 \% \mathrm{v} / \mathrm{v})$ diluted in PBS for $10-\mathrm{min}$, followed by a 5-min wash in PBS for 3 times.

2. Prepare blocking buffer as Recipe 5. Centrifuge the blocking buffer at $16,000 \times g$ for $10 \mathrm{~min}$ in case there was any residue precipitate from goat serum. Incubate fibers with $400 \mu$ l blocking solution for $1 \mathrm{~h}$ at room temperature.

3. Incubate with $200 \mu \mathrm{l}$ blocking buffer containing primary antibodies of Pax7 (1:100) or MyoD (1:300) overnight at $4{ }^{\circ} \mathrm{C}$.

4. After night incubation with primary antibody, wash myofibers 3 times for 5-min with PBS containing $0.5 \%$ Tween 20 to remove any unbound antibody.

5. Incubate with $200 \mu$ l blocking buffer containing appropriate secondary antibody (1:500) for 1-h at room temperature protected from light.

6. After 1-h incubation with secondary antibody, wash 3 times at 5 min per wash with PBS containing $0.5 \%$ Tween 20 .

7. Transfer each fiber to a glass slide and drain the remaining liquid.

8. Mount the glass slide with DAPI-containing mounting medium. Cover with a cover slide and dry the glass slide for at least 10 min before imaging.

9. Observe myofibers under a fluorescent microscope and capture images of immunostained myofibers (Figure 4). 


\section{bïo-protocol

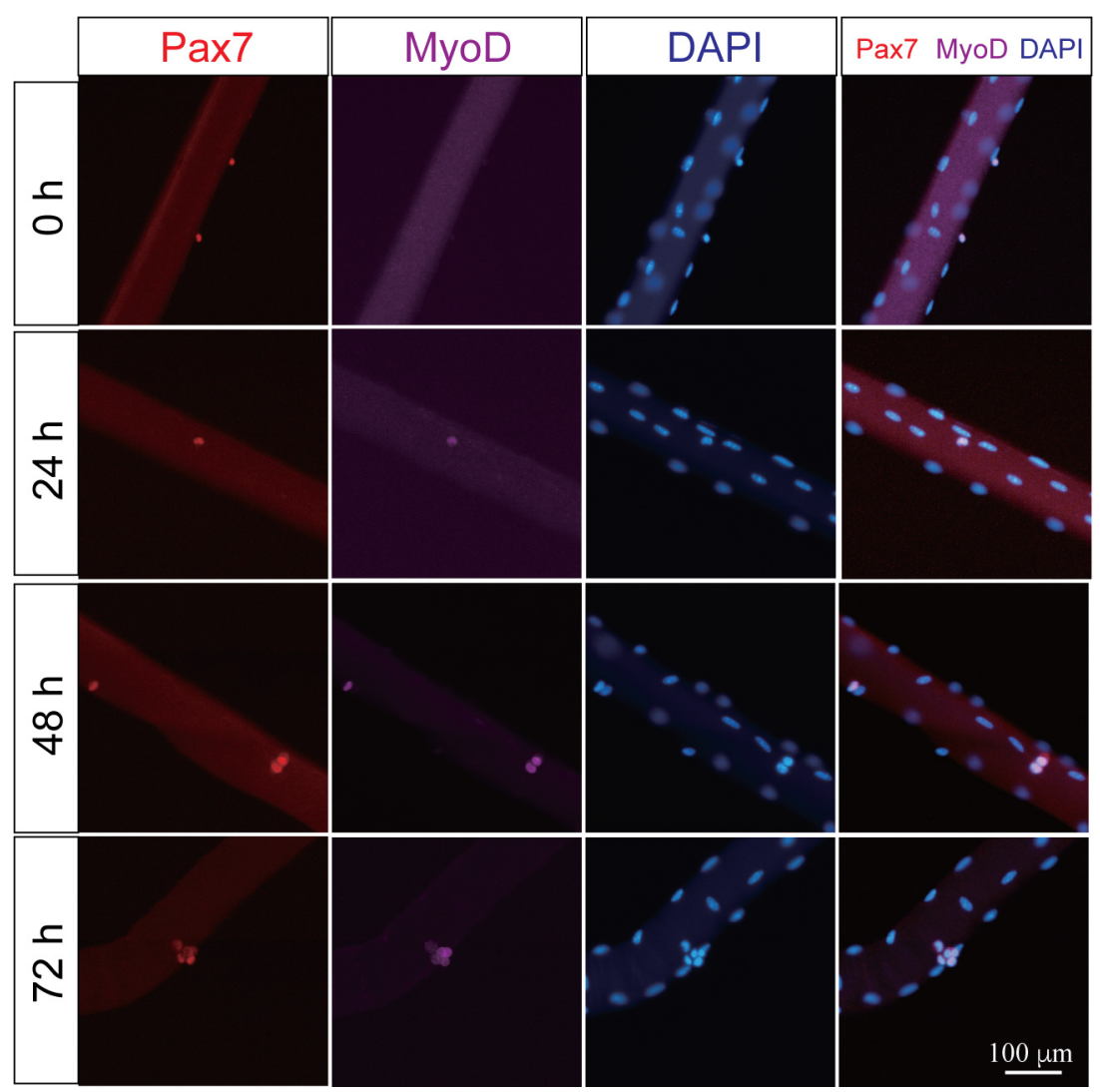

Figure 4. Representative images of single myofiber culture harvested at T0, 24, 48 and 72-h. Single myofiber with satellite cells or clusters were fixed and immunostained with antibodies against Pax7 or MyoD. Red for $\mathrm{Pax}^{+}$satellite cells. Purple for $\mathrm{MyoD}^{+}$satellite cells. Blue for nucleus. Scale bar $=100 \mu \mathrm{m}$.

\section{Data analysis}

Based on our protocol, around $\sim 200$ myofibers could be isolated from each EDL muscle. The average number of $\mathrm{Pax}^{+}$satellite cells on each myofiber at T0 is 6-8 and the $\mathrm{MyoD}^{+}$satellite cells could be barely detected at T0. After 24-h culture, the number of $\mathrm{MyoD}^{+}$satellite cells is increased upon satellite cells activation. Proliferated satellite cells could be observed at 48-h timepoint. The satellite cells cluster could be observed after 72-h culture (Figure 4).

\section{$\underline{\text { Notes }}$}

1. Avoid bubbles when coating plates with horse serum.

2. Do not let tweezers or scissors touch the EDL muscle.

3. When using the bent plastic dropper to transfer muscle fiber, single myofiber should be inhaled along one end of the muscle fiber to avoid snapping the muscle.

4. When transferring myofibers, insert the nozzle below the liquid level to prevent the muscle fibers from being exposed to air. 
5. Avoid bubbles when covering glass slips.

\section{Recipes}

1. Washing media

DMEM supplemented with:

$10 \%$ fetal bovine serum

$2 \%$ sodium pyruvate

$1 \%$ Penicillin-Streptomycin

2 mM EDTA

Filter the solution through a $0.2 \mu \mathrm{m}$ filter (optional, to remove any debris from serum)

2. Collagenase solution

DMEM supplemented with $0.2 \%$ Collagenase Type 1

3. $4 \%$ paraformaldehyde

a. Dissolve $4 \mathrm{~g}$ paraformaldehyde powder in $100 \mathrm{ml}$ PBS.

b. Heat the solution to $65^{\circ} \mathrm{C}$ until paraformaldehyde completely dissolved

c. Filter the solution through a $0.2 \mu \mathrm{m}$ filter and store at $-20^{\circ} \mathrm{C}$

4. Culture media

DMED supplemented with $20 \%$ fetal bovine serum

$1 \%$ chicken embryo extract

$1 \%$ Penicillin-Streptomycin

Filter the solution through a $0.2 \mu \mathrm{m}$ filter

5. Blocking buffer

PBS supplemented with:

$3 \%$ BSA

$0.5 \%$ Tween 20

$5 \%$ goat serum (added right before the blocking procedure)

\section{Acknowledgments}

This work was support by 'GDAS' Project of Science and Technology Development (Grant No. 2018GDASCX-0806) to Liwei Xie.

Author contribution: LW. X. designed the experiment and collected the grant for present study. LW. X., SJ. C., HR. D. and XP. Y. developed and optimized the methodology. SJ. C. collected and analyzed the data. SJ. C. drafted the manuscript and LW. X. proofread the manuscript.

\section{Competing interests}

The authors declare that there is no conflict of financial or research interest. 


\section{Ethics}

C57BL/6J mice were raised at the SPF animal facility of Guangdong Institute of Microbiology (GDIM) in a $12 / 12$ dark-light cycle with ad lithium free access to food and water. The animal protocol was proved by the Institute Animal Care Use Committees of GDIM (Permission \#: GT-IACUC201704071).

\section{References}

1. Bekoff, A. and Betz, W. (1977a). Properties of isolated adult rat muscle fibres maintained in tissue culture. J Physiol 271(2): 537-547.

2. Bekoff, A. and Betz, W. J. (1977b). Physiological properties of dissociated muscle fibres obtained from innervated and denervated adult rat muscle. J Physiol 271(1): 25-40.

3. Bischoff, R. (1986). Proliferation of muscle satellite cells on intact myofibers in culture. Dev Biol 115(1): 129-139.

4. Collins, C. A., Olsen, I., Zammit, P. S., Heslop, L., Petrie, A., Partridge, T. A. and Morgan, J. E. (2005). Stem cell function, self-renewal, and behavioral heterogeneity of cells from the adult muscle satellite cell niche. Cell 122(2): 289-301.

5. Danoviz, M. E. and Yablonka-Reuveni, Z. (2012). Skeletal muscle satellite cells: background and methods for isolation and analysis in a primary culture system. Methods Mol Biol 798: 2152.

6. Gallot, Y. S., Hindi, S. M., Mann, A. K. and Kumar, A. (2016). Isolation, culture, and staining of single myofibers. Bio-protocol 6(19): e1942.

7. Lim, C. L., Ling, K. H. and Cheah, P. S. (2018). Isolation, cultivation and immunostaining of single myofibers: An improved approach to study the behavior of satellite cells. $J$ Biol Methods 5(1): e87.

8. Mauro, A. (1961). Satellite cell of skeletal muscle fibers. J Biophys Biochem Cytol 9: 493-495.

9. Pasut, A., Jones, A. E. and Rudnicki, M. A. (2013). Isolation and culture of individual myofibers and their satellite cells from adult skeletal muscle. $J$ Vis $\operatorname{Exp(73):~e50074.~}$

10. Rosenblatt, J. D., Lunt, A. I., Parry, D. J. and Partridge, T. A. (1995). Culturing satellite cells from living single muscle fiber explants. In Vitro Cell Dev Biol Anim 31(10): 773-779. 